\section{Avaliação da integralidade: conferindo sentido para os pactos na programação de metas dos sistemas municipais de saúde}

\author{
Evaluation of comprehensiveness: \\ ascribing meaning to agreements on goals \\ for local health systems
}

\author{
1 Departamento de Saúde \\ Pública, Universidade \\ Federal de Santa Catarina, \\ Florianópolis, Brasil. \\ Correspondência \\ E. M. Conill \\ Rua Vento Sul 306, \\ Florianópolis, $S C$ \\ 88063-070, Brasil. \\ eleonorc@bol.com.br
}

\begin{abstract}
As a policy guideline, ideal, or objective, comprehensiveness is a relevant attribute in the evaluation of quality of care and health systems. The article discusses the meaning of the concept of comprehensiveness in practice in some health services and systems, analyzing various methodologies to implement the concept. In Brazil, what prevails is an expanded perception emphasizing the complete, continuous, and coordinated nature of health care and management. The article highlights the importance of combining the evaluation of comprehensiveness with measures of access, suggesting the selection of indicators pertaining to agreements on goals for local health systems and their monitoring. This initiative could enhance the validity of existing evaluation instruments, from a communications perspective.
\end{abstract}

Comprehensive Health Care; Health Care Quality Assessment; Delivery of Health Care

\section{Introdução}

Houve um saudável avanço nos mecanismos e instrumentos de acompanhamento e avaliação utilizados pelas instâncias gestoras do Sistema Único de Saúde (SUS), quando comparados com as antigas práticas das instituições federais, centradas na revisão de contas médicas e no cumprimento de metas de produção ou de metas de programas verticais. Os sistemas de informações também se diversificaram disponibilizando um leque amplo de dados que tendem a ser mais integrados por meio desse instrumental. Ainda que sob forte regulação federal, nota-se a busca em estabelecer instâncias de negociação entre os gestores, com um planejamento de tipo mais comunicativo.

$\mathrm{O}$ argumento deste texto é de que um passo importante a ser dado seria o monitoramento de algumas das diretrizes básicas da política de saúde na prestação dos serviços, entre elas, o princípio da integralidade da atenção. A idéia é de que isso possa conferir, às avaliações do desempenho de metas pactuadas nos municípios, um sentido mais amplo de avaliação da qualidade dos sistemas municipais.

Conforme veremos, os mesmos aspectos que envolvem a avaliação de serviços e dos sistemas de saúde se aplicam ao esforço para avaliar a integralidade, ou seja: a multifatorialidade do processo saúde/doença, a diferença entre a dimensão individual e coletiva do concei- 
to de qualidade, a relatividade da noção de valor e o fato de que os processos avaliativos lidam com relações sociais e processos organizacionais complexos.

O objetivo de garantir serviços de saúde integrais surge com a expansão das políticas sociais e dos sistemas de saúde na primeira metade do século XX. No âmbito das democracias ocidentais, a criação do National Health Servi$c e$ (NHS), no Reino Unido, é o exemplo mais expressivo desse ideal regulatório nas políticas de saúde.

Em 1946, o NHS Act estabeleceu o princípio da responsabilidade coletiva por serviços completos (comprehensive health services), que deveriam ser disponíveis e gratuitos para toda a população. Alguns anos mais tarde, em meados da década de 60, esse objetivo reaparece nas reformas setoriais, de forma mais contundente naquela realizada nos serviços sociais e de saúde da província do Québec, em 1971, reforma Castonguay-Nepveu, na qual o acesso universal e a integralidade, "la gobalité des soins", apareciam como centrais.

No Brasil, a integralidade fez parte das propostas de reforma desde o início da década de 80, surgiu em programas abrangentes para grupos específicos (Programa de Atenção Integral à Saúde da Mulher - PAISM -, Programa de Atenção Integral à Saúde da Criança - PAISC), sendo finalmente assumida como diretriz para a organização do SUS. No atual Programa de Expansão e Consolidação da Saúde da Família (PROESF - http://dtr2002.saude.gov.br/proesf/, acessado em 27/Abr/2004), a atenção básica é considerada como sendo estratégica para a conversão do modelo de atenção nos grandes centros urbanos e para assegurar um cuidado integral à saúde da população.

Diretriz política, ideal ou objetivo, a integralidade torna-se um atributo relevante a ser levado em conta na avaliação da qualidade do cuidado, dos serviços e dos sistemas de saúde, em geral, naqueles direcionados para uma política de cuidados primários.

Três grupos de questões podem ser circunscritos nesse sentido: (1) qual é o entendimento acerca desse conceito e como verificar sua expressão prática?; (2) quais modelos de atenção propiciariam processos de trabalho favorecedores de ações mais integrais e qual a influência dos fatores contextuais sobre esses processos?; e (3) quais os efeitos de um cuidado, serviço ou sistema integral sobre a situação da saúde?

O segundo grupo relaciona-se, de forma mais direta, com a área da organização e da gestão dos serviços, inserindo-se no campo da avalia- ção no que vem sendo denominado de análise estratégica e de análise de implantação. A primeira explora a pertinência de uma intervenção analisando sua adequação à situação social que lhe deu origem, e a segunda avalia a influência do contexto e da variação no grau de implantação sobre os efeitos esperados 1. Estudos avaliando a implantação da diretriz de integralidade em suas relações contextuais e organizacionais são ainda escassos, o mesmo ocorrendo com aqueles que se referem aos resultados de modelos mais integrais 2,3,4,5.

Responder as questões do primeiro grupo representa o desafio de transformar um princípio no campo das políticas em categoria operativa no campo da avaliação. Constituem prérequisito para a realização de qualquer tipo de pesquisa avaliativa, além de necessárias para o monitoramento ou reflexão acerca da qualidade no quotidiano dos serviços que pretendam ser coerentes com essa política.

Essa é a razão pela qual optamos por enfocar, neste trabalho, o significado atribuído a esse conceito na prática de alguns serviços e sistemas de saúde, analisando metodologias utilizadas ou sugeridas para avaliá-lo. Na conclusão, correlacionamos esse aporte com a gestão do SUS, sintetizando tópicos que podem ser levados em conta para aumentar a validade do instrumental hoje utilizado para o acompanhamento e avaliação dos sistemas municipais de saúde, inserindo-o numa perspectiva comunicativa e dialógica.

\section{Concepções e metodologias na operacionalização de um atributo complexo}

No Reino Unido, a preocupação em operacionalizar esse atributo não tem sido predominante, dando lugar às avaliações que priorizam o acesso. A redução das listas e dos tempos de espera continuava encabeçando as prioridades para o período 1999-2002, na atual reforma do NHS 6 . Há também ênfase na prevenção de doenças coronarianas e neoplasias, saúde mental, serviços comunitários e atenção ao idoso, demonstrando a preocupação com a manutenção de um leque completo de serviços.

$\mathrm{Na}$ Inglaterra, foram criadas novas estruturas administrativas - os Primary Care Trusts (PCTs), responsáveis pela gestão dos Distritos de Saúde, incluindo a integração entre a saúde pública e os generalistas, e pela compra dos demais tipos de serviços. Em pesquisa acerca da qualidade da atenção primária, promovida pelo PCT do bairro de Islington em Londres 7, foi possível observar a 
percepção e a expressão da integralidade em nível local, com base na opinião de 649 usuários complementada com entrevistas junto à equipe de gestão e análise de documentos.

É grande a gama de serviços ofertados, havendo preocupação em ampliá-la ainda mais disponibilizando, por exemplo, coleta mais rápida de exames laboratoriais, apoio de especialista em diabetes, nutrição, fisioterapia a serem compartilhados entre os consultórios. Cogitase, também, em oferecer informações referentes a benefícios sociais nos consultórios. No entanto, apesar da noção de uma cobertura integral ter sido introduzida e vir sendo ampliada no NHS, a avaliação concentra-se no acesso através de medidas dos tempos de espera e da eqüidade entre grupos sociais, principalmente das minorias étnicas. A oferta é ampla, mas interessa à sociedade saber a quem ela está beneficiando e quanto tempo leva-se para obtê-la.

No Québec, um estudo da expressão do princípio de "globalité" foi realizado pela observação dos cuidados prestados nos Centres Locaux de Services Communautaires (CLSC), porta de entrada do novo sistema de saúde. O substrato legal e normativo da reforma Castonguay-Nepveu serviu para operacionalizar esse princípio, identificando-se cinco dimensões: a dimensão pessoal e familiar do cuidado, seu caráter completo (gama de serviços), contínuo (seguimento no tempo, no domicílio e no sistema) e coordenado (relações entre os serviços). Essas dimensões foram avaliadas com indicadores específicos, obtidos através de observação direta, entrevistas e documentos 2 .

O fornecimento de cuidados integrais nos CLSCs foi a inovação de maior expressão encontrada nessa reforma, demonstrando-se a existência de duas tendências nas práticas. De um lado, um entendimento ampliado significando a incorporação de ações preventivas, sociais e comunitárias, com introdução de ações da esfera da saúde coletiva nos serviços tradicionalmente dominados pelo cuidado médico. Por outro, representava a garantia de maior continuidade de uma atenção médica de primeira linha, agora mais completa. No decorrer dos anos e nas sucessivas reformas operadas nessa Província, a avaliação e discussão da "globalité" parece ter perdido terreno para outras temáticas 8 .

Na literatura internacional, Starfield ${ }^{9}$ sugeriu um modelo pioneiro para avaliar a atenção primária. Propôs que isso fosse feito a partir da análise da estrutura e dos processos de atenção, tomando-se por base quatro elementos principais: o atributo do primeiro contato, o caráter coordenado ou integrador (seguimento ou continuidade do atendimento), o caráter amplo ou integral (oferta completa de serviços bio-psico-sociais, referência) e a "longitudinalidade" (vínculo no tempo).

O entendimento da integralidade enquanto um dos atributos da atenção primária, medida por meio dos serviços ofertados, tem predominado em estudos mais recentes da mesma autora ou de outros pesquisadores americanos.

Esse critério foi utilizado por Larizgoitia \& Starfield 3 para avaliar a reforma da atenção primária na Espanha, verificando-se a realização de atividades preventivas (medida da tensão arterial, vacinação contra influenza e realização de mamografia) na região Basca. Em outro trabalho 10 acerca das diferenças na qualidade da atenção primária no "managed care" americano, a integralidade foi vista através da lista de serviços disponíveis, referidos por prestadores e usuários.

Franks et al. 11 utilizaram-na para identificar se a definição de atenção primária estabelecida pelo Instituto de Medicina dos Estados Unidos expressava-se no exercício de algumas especialidades (1985-1991), avaliando-a através da existência do primeiro contato e da continuidade do atendimento ao longo do ciclo vital. Confundiu-se, nesse caso, com os atributos de acesso e "longitudinalidade" (vínculo), o que demonstra a diversidade de interpretações.

Em nosso meio, Carvalho 12 realizou um dos primeiros estudos após a implantação do SUS, por meio da análise de uma área programática do Rio de Janeiro, Brasil. Utilizou um inventário da gama de atividades, considerando-a como o "conjunto articulado de promoção, proteção, recuperação e reabilitação” 12 (p. 35).

Mais recentemente, Ortiga 13 enfocou os efeitos da descentralização no acesso e na integralidade da atenção dos sistemas municipais, através da presença de programas preventivos e inovadores (imunização, câncer cérvico-uterino, odontologia, o Programa Saúde da Família - PSF). Complementou o estudo com a percepção dos gestores, profissionais e conselheiros de saúde.

Utilizamos o acesso e a integralidade como categorias operativas ao avaliar a implantação do PSF em Florianópolis, Santa Catarina, Brasil, no período 1994-2000, selecionando variáveis da estrutura e do processo de atenção. Optamos pela análise do caráter completo do cuidado (gama de serviços ofertados) e de sua continuidade (referência, visitas domiciliares), usando observação direta, entrevistas e dados provenientes do Sistema de Informação da Atenção Básica (SIAB) 14.

Nesse trabalho, as coordenações dos postos referiram-se à integralidade como um conjun- 
to amplo de ações, um acompanhamento ampliado e diferenciado dos indivíduos, não apenas consulta.

Esse é o mesmo entendimento de um painel de experts consultados para validação de um instrumento visando avaliar a integralidade nos sistemas municipais, desenvolvido por Giovanella et al. 15. Nesse caso, o enfoque foi o processo de gestão e o funcionamento de estruturas facilitadoras da assunção da responsabilidade integral.

Tomou-se por base um conceito composto por quatro dimensões, correspondendo a campos distintos de intervenção: (1) primazia das ações de promoção e prevenção (campo da política de prioridades); (2) garantia de atenção nos três níveis de complexidade da assistência médica (campo da organização da atenção); (3) articulação das ações de promoção, prevenção e recuperação (campo da gestão); (4) abordagem integral do indivíduo (campo do cuidado individual).

Dos 30 critérios distribuídos entre essas dimensões, 24 foram considerados muito relevantes, e 17, bastante aplicáveis, mas o conjunto foi considerado insuficiente. Os entrevistados propuseram a utilização de informações provenientes do pacto do Piso Assistencial Básico (PAB) e da Programação Pactuada e Integrada de Epidemiologia e Controle de Agravos (PPI-ECD) e de alguns dos sistemas de informações vigentes.

A incorporação do instrumental já existente coincide com as diretrizes formuladas pelo Ministério da Saúde para orientar o desenvolvimento de estudos avaliativos, denominados de linha de base, do PROESF, sugerindo-se o uso de dados provenientes da PPI (assistência, epidemiologia e vigilância sanitária), do Pacto de Indicadores da Atenção Básica e do SIAB (http://dtr2002.saude.gov.br/proesf/, acessado em 27/Abr/2004).

A partir da análise desses trabalhos, é possível sintetizarmos (Tabela 1) os principais aspectos, opções e dilemas relacionados com a operacionalização desse atributo, quais sejam: análise de poucas ou múltiplas dimensões, uso de outros atributos associados, dados provenientes de fontes primárias ou secundárias, da opinião de gestores, profissionais ou dos usuários, e decisão se o enfoque será centrado no sistema, no cuidado individual ou em ambos. Vejamos alguns tópicos que devem ser levados em conta nessas escolhas.

\section{Discussão e conclusão: sobre a relação entre acesso e integralidade e a necessidade da diversificação do olhar sem excesso de informações}

No caso do Brasil, parece predominar uma percepção ampliada da diretriz da integralidade, significando, além da gama de serviços, seu caráter contínuo e coordenado. Nota-se também a preocupação em enfocar os cuidados e as condições de gestão que o determinam.

É possível que isso esteja relacionado com a importância de garantir-se a difusão de um novo modelo de organização de serviços em situações de aquisição e manutenção de novos direitos sociais, o que coincide com o ocorrido nos momentos iniciais da reforma do Québec. Há, também, uma tendência generalizada em considerar as práticas da atenção básica como um lócus privilegiado para observar a expressão desse princípio.

Outro aspecto observado é que a avaliação da integralidade vem associada ou mesmo confundida com medidas de acesso. Um conjunto amplo de ações ofertado num sistema de saúde só terá efetividade na medida de sua utilização com eqüidade. Da mesma forma, não interessa ter acesso a cuidados parcelares e descontínuos. Ou seja, na realidade, o que vale a pena verificar é se está ocorrendo acesso a um sistema com cuidados integrais.

A verificação das dimensões físicas do acesso, ou seja, da existência de recursos capazes de responder a demanda, dá também uma idéia das condições estruturais para que a integralidade possa ocorrer. No estudo do caso da implantação do PSF em Florianópolis, foram detectados problemas em decorrência do quantitativo insuficiente de recursos humanos e na referência para serviços especializados. Concluiu-se que essa barreira poderia comprometer vantagens obtidas na integralidade ao impedir a realização de certas atividades, tais como, a prevenção e as visitas domiciliares, por sobrecarga da demanda por atenção curativa. Havendo problemas na referência para especialidades, a continuidade tornava-se também difícil, e a qualidade prejudicada como um todo.

Avaliar conjuntamente o acesso e a integralidade exige esforços suplementares em sua operacionalização com escolha de informações que sejam viáveis de serem coletadas, válidas, mas não numerosas.

A validade de um instrumento de avaliação refere-se à sua adequação ao conceito teórico ou objeto que se quer medir ou intervir, sendo estimada através da validade de conteúdo (conceitos adequados), da validade do critério de 
Avaliação da integralidade: síntese de metodologias.

\begin{tabular}{|c|c|c|c|c|c|c|}
\hline Autor & Ano & Objetivo/Contexto & Unidade de análise & Dimensões & Outros atributos & Fontes/Instrumentos \\
\hline Conill 2 & 1982 & $\begin{array}{l}\text { Saúde comunitária/ } \\
\text { Reforma do Québec }\end{array}$ & Centros de Saúde & $\begin{array}{l}\text { Cuidado pessoal e } \\
\text { familiar; completo; } \\
\text { contínuo; coordenado }\end{array}$ & Acesso; participação & $\begin{array}{l}\text { Documentos; entrevistas; } \\
\text { observação direta }\end{array}$ \\
\hline Carvalho 12 & 1991 & $\begin{array}{l}\text { Modelos assistenciais/ } \\
\text { Reforma brasileira }\end{array}$ & Unidades Básicas & $\begin{array}{l}\text { Completo; contínuo; } \\
\text { vínculo }\end{array}$ & $\begin{array}{l}\text { Atividades interativas; } \\
\text { ensino; pesquisa; } \\
\text { planejamento }\end{array}$ & $\begin{array}{l}\text { Documentos, entrevistas; } \\
\text { observação direta }\end{array}$ \\
\hline $\begin{array}{l}\text { Larizgoitia \& } \\
\text { Starfield } 3\end{array}$ & 1997 & $\begin{array}{l}\text { Atenção primária/ } \\
\text { Reforma da Espanha }\end{array}$ & Usuários & Completo & $\begin{array}{l}\text { Acessibilidade; } \\
\text { longitudinalidade; } \\
\text { vínculo; qualidade } \\
\text { técnica }\end{array}$ & Inquérito domiciliar \\
\hline Franks et al. 11 & 1997 & $\begin{array}{l}\text { Atenção primária/ } \\
\text { Estados Unidos }\end{array}$ & Consultas médicas & $\begin{array}{l}\text { Primeiro contato; } \\
\text { vínculo }\end{array}$ & $\begin{array}{l}\text { Coordenação; } \\
\text { continuidade; } \\
\text { acessibilidade }\end{array}$ & $\begin{array}{l}\text { Inquérito nacional } \\
\text { com médicos }\end{array}$ \\
\hline $\begin{array}{l}\text { Starfield } \\
\text { et al. } 10\end{array}$ & 1998 & $\begin{array}{l}\text { Atenção primária/ } \\
\text { "managed care" } \\
\text { americano }\end{array}$ & Usuários, médicos & Cuidado completo & $\begin{array}{l}\text { Acessibilidade; } \\
\text { vínculo; coordenação; } \\
\text { familiar; comunitário; } \\
\text { culturalmente } \\
\text { adequado }\end{array}$ & $\begin{array}{l}\text { Inquérito (telefone } \\
\text { e correio) }\end{array}$ \\
\hline Ortiga 13 & 1999 & Descentralização/SUS & Município & $\begin{array}{l}\text { Caráter completo } \\
\text { das ações }\end{array}$ & Acesso & $\begin{array}{l}\text { Documentos de } \\
\text { planejamento e gestão; } \\
\text { sistemas de informações; } \\
\text { entrevistas }\end{array}$ \\
\hline Conill 14 & 2002 & Atenção primária/PSF & Equipes, famílias & $\begin{array}{l}\text { Cuidado completo; } \\
\text { contínuo }\end{array}$ & Acesso & $\begin{array}{l}\text { Entrevistas, observação } \\
\text { direta; SIAB }\end{array}$ \\
\hline $\begin{array}{l}\text { Giovanella } \\
\text { et al. } 15\end{array}$ & 2002 & Gestão municipal/SUS & Município & \multicolumn{2}{|l|}{$\begin{array}{l}\text { Promoção; prevenção; } \\
\text { atenção médica completa; } \\
\text { articulação das ações; } \\
\text { cuidado individual }\end{array}$} & $\begin{array}{l}\text { Documentos de } \\
\text { planejamento e gestão; } \\
\text { sistemas de informações }\end{array}$ \\
\hline
\end{tabular}

predição (sensibilidade, especificidade) e da validade de construção (operacionalização) 16. É importante ter em mente a distinção entre sistema de saúde e subsistema de atenção, uma vez que a integralidade é uma diretriz operacional da política de saúde que deve expressarse na estrutura e nos processos do segundo 17.

Pelo menos, dois instrumentos hoje existentes foram citados e podem contribuir para a obtenção de informações secundárias acerca do caráter completo, contínuo e coordenado das ações nos sistemas municipais. São eles, o Pacto dos Indicadores da Atenção Básica e a Programação Pactuada e Integrada (PPI), tanto a da assistência como àquela referente à epidemiologia e à vigilância sanitária. Esses mesmos instrumentos fornecem indicadores de morbidade e mortalidade, podendo permitir que, no futuro, possamos pensar em prospecções acerca de medidas do impacto da integralidade.
Uma interface mais profícua entre a pesquisa avaliativa e aquela feita de forma rotineira nos serviços poderia facilitar a seleção de indicadores já validados.

O MS vem demonstrando interesse em promover o que denomina de institucionalização da avaliação, através da continuidade de pesquisas já realizadas e da ampliação do leque de metodologias (http://dtr2002.saude.gov.br/ proesf/, acessado em 27/Abr/2004).

Avaliar significa a realização de um julgamento de valor, o que implica no reconhecimento da existência de diferentes olhares sobre uma mesma realidade. Isso reforça a necessidade de coerência entre as iniciativas já existentes na direção de um planejamento de tipo comunicativo com a promoção de organizações dialógicas também no campo da avaliação 18. Ainda que seja ampliado o leque de metodologias a serem utilizadas, é preciso cautela 
para que não haja excesso de indicadores com uma externalidade acadêmica ou tecnocrática na coleta de informações.

A sustentação teórica da importância e das possibilidades que podem ser realizadas nesse sentido escapa ao escopo deste trabalho, mas pode ser obtida com profundidade nos trabalhos desenvolvidos por Rivera 19. Também seriam úteis a difusão e adequação, no Brasil, da abordagem de avaliação participativa desenvolvida por Chambers 20,21 para projetos rurais de países em desenvolvimento, a qual vem sendo aplicada com sucesso na área da saúde em áreas urbanas de diversos países. Fortemente ancorada em técnicas qualitativas e visuais, essa abordagem inicia-se com o levantamento de temáticas chaves até chegar-se a sua verificação com propostas de solução, buscando-se uma simetria cultural na coleta e na análise das informações. Seu uso poderia facilitar a obtenção de informações primárias complementares provenientes dos usuários.
No movimento de institucionalizar a avaliação, é possível pensar na promoção de consensos acerca do significado e dos indicadores considerados centrais para avaliar a integralidade, tanto no âmbito da gestão do sistema municipal como no dos serviços, caminhandose, assim, para uma maior validade de conteúdo e de construção em torno do instrumental já existente.

Embora o conceito de avaliação enfatize a questão do juízo de valor, a preocupação operacional continua ainda predominante, fazendo com que seja a medida, e não o juízo, a questão até agora mais desenvolvida.

Esses consensos ou discussões podem vir a conferir um sentido ampliado para a análise do desempenho das metas pactuadas, inserindoas numa perspectiva mais integrada e participativa de avaliação da qualidade dos sistemas municipais de saúde. Para além da necessária exigência administrativa de regular ou assegurar repasses de recursos, é preciso não perder de vista os princípios fundamentais que orientam a política de saúde.

\section{Resumo}

Diretriz política, ideal ou objetivo, a integralidade torna-se um atributo relevante na avaliação da qualidade do cuidado e dos sistemas de saúde. Discute-se o significado desse conceito na prática de alguns serviços e sistemas de saúde, analisando metodologias para sua operacionalização. No Brasil, predomina uma percepção ampliada com ênfase no caráter completo, contínuo e coordenado do cuidado e da gestão. Destaca-se a importância de associar a avaliação da integralidade com medidas de acesso, sugerindo-se a escolha de indicadores dos pactos de metas das programações municipais para seu monitoramento. Tal iniciativa poderia aumentar a validade do instrumental de avaliação existente, numa perspectiva comunicativa. 


\section{Referências}

1. Denis JL, Champagne F. Análise da implantação In: Hartz ZMA, organizador. Avaliação em saúde. Dos modelos conceituais à prática na análise da implantação de programas. Rio de Janeiro: Editora Fiocruz; 1997. p. 49-83.

2. Conill EM. Les implications de la santé communautaire en tant que nouvelle politique de santé: analyse du cas du Québec [PhD Thesis]. Paris: Institut d'Étude du Développement Economique et Social, Université de Paris I/Sorbonne; 1982.

3. Larizgoitia J, Starfield B. Reform of primary care: the case of Spain. Health Policy 1997; 41:355-65.

4. Safran DG, Tarlov AR, Rogers WH. Primary care performance in fee for service and prepaid health care systems. JAMA 1994; 271:1579-86.

5. Starfield B, Shi L. Policy relevant determinants of health; an international perspective. Health Policy 2002; 60:201-8.

6. European Observatory on Health Systems and Policies. Health care systems in transition. Copenhagen: WHO Regional Office for Europe; 1999.

7. Health Ideas Islington. Health services in Islington. What the people think, their experiences and views of GP's and other primary health care. London: Manor Gardens Centre; 2003.

8. Conill EM. A recente reforma dos serviços de saúde na província do Québec, Canadá: as fronteiras da preservação de um sistema público. Cad Saúde Pública 2000; 16:963-71.

9. Starfield B. Measuring the attainment of primary care. J Med Educ 1979; 54:361-9.

10. Starfield B, Cassady C, Namda J, Forrest CB, Berk, R. Consumer experiences and provider perceptions of the quality of primary care: implications for managed care. J Fam Pract 1998; 46:216-25.

11. Franks, P, Clancy CM, Nutting PA. Defining primary care. Empirical analysis of the National Ambulatory Medical Care Survey. Med Care 1997; 35:655-68.
12. Carvalho MR. Modelos assistenciais de unidades básicas e integralidade. Estudo de caso: a área de planejamento 3.1 (RJ/RJ), contribuição para sua distritalização [Dissertação de Mestrado]. Rio de Janeiro: Escola Nacional de Saúde Pública, Fundação Oswaldo Cruz; 1991.

13. Ortiga AMB. Efeitos da municipalização no acesso e na integralidade dos serviços de saúde, Blumenau, SC, (1994-1998) [Dissertação de Mestrado]. Florianópolis: Departamento de Saúde Pública, Universidade Federal de Santa Catarina; 1999.

14. Conill EM. Políticas de atenção primária e reformas sanitárias: discutindo a avaliação a partir da análise do Programa Saúde da Família em Florianópolis, Santa Catarina, Brasil, 1994-2000. Cad Saúde Pública 2002; 18 Suppl:191-202.

15. Giovanella L, Lobato LVC, Carvalho AI, Conill EM, Cunha EM. Sistemas municipais de saúde e a diretriz da integralidade da atenção: critérios para avaliação. Saúde em Debate 2002; 26:37-61.

16. Contandriopoulos AP, Champagne F, Potvin L, Denis JL, Boyle P. Saber preparar uma pesquisa. São Paulo: Editora Hucitec/Rio de Janeiro: ABRASCO; 1994.

17. Hortale VA, Conill EM, Pedroza M. Desafios na construção de um método para análise comparada da organização de serviços de saúde. Cad Saúde Pública 1999; 15:79-88.

18. Rivera FJU, Artmann E. Planejamento e gestão em saúde: flexibilidade metodológica e agir comunicativo. Ciênc Saúde Coletiva 1999; 4:355-65.

19. Rivera FJU. Análise estratégica em saúde e a gestão pela escuta. Rio de Janeiro: Editora Fiocruz; 2003.

20. Chambers R. The origins and practice of participatory rural appraisal. World Dev Forum 1994; 22:953-69.

21. Chambers R. Participatory rural appraisal (PRA): analysis of experience. World Dev Forum 1994; 22:1253-68.

Recebido em 14/Jun/2004 Aprovado em 15/Jun/2004 